\title{
Epidemiology of Strongyloides stercoralis in northern Italy: results of a multicentre case-control study, February 2013 to July 2014
}

D Buonfrate ${ }^{1}$, M Baldissera ${ }^{2}$, F Abrescia ${ }^{3}$, M Bassetti 4 , G Caramaschi 5 , M Giobbia ${ }^{6}$, M Mascarello ${ }^{7}$, P Rodari ${ }^{8}$, N Scattolo 9

, G Napoletano ${ }^{2}$, Z Bisoffi ${ }^{1}$, on behalf of the CCM Strongyloides Study Group ${ }^{10}$

1. Centre for Tropical Diseases, Sacro Cuore Hospital, Negrar (Verona), Italy

2. Prevention Department, ULSS20, Verona, Italy

3. Medici per la Pace Onlus, Verona, Italy

4. University Hospital of Udine, Udine, Italy

5. Servizio di Medicina di Laboratorio, Azienda Ospedaliera Carlo Poma, Mantova, Italy

6. Infectious Diseases Department, Ca' Foncello Hospital, Treviso, Italy

7. Department of Infectious Diseases, University Hospital of Trieste, Trieste, Italy

8. University Department of Infectious and Tropical Diseases, University of Brescia and Spedali Civili General Hospital, Brescia, Italy

9. Laboratorio Analisi, Ospedale Fracastoro, San Bonifacio (Verona), Italy

10. Members of the group are listed at the end of the article

Correspondence: Dora Buonfrate (dora.buonfrate@sacrocuore.it)

Citation style for this article:

Buonfrate D, Baldissera M, Abrescia F, Bassetti M, Caramaschi G, Giobbia M, Mascarello M, Rodari P, Scattolo N, Napoletano G, Bisoffi Z, on behalf of the CCM Strongyloides Study Group. Epidemiology of Strongyloides stercoralis in northern Italy: results of a multicentre case-control study, February 2013 to July 2014. Euro Surveill. 2016;21(31):pii=30310. DOI: http://dx.doi.org/10.2807/1560-7917.ES.2016.21.31.30310

Article submitted on 16 October 2015 / accepted on 18 February 2016 / published on 04 August 2016

Strongyloides stercoralis is a soil-transmitted helminth widely diffused in tropical and subtropical regions of the world. Autochthonous cases have been also diagnosed sporadically in areas of temperate climate. We aimed at defining the epidemiology of strongyloidiasis in immigrants and Italians living in three northern Italian Regions. Screening for $S$. stercoralis infection was done with serology, confirmation tests were a second serological method or stool agar culture. A case-control approach was adopted and patients with a peripheral eosinophil count $\geq 500 / \mathrm{mcL}$ were classified as cases. Of 2,701 individuals enrolled here 1,351 were cases and 1,350 controls; $86 \%$ were Italians, $48 \%$ women. Italians testing positive were in $8 \%(97 / 1,137)$ cases and $1 \%(13 / 1,178)$ controls (adjusted odds ratio (aOR) 8.2; 95\% confidence interval (Cl): 4.5-14.8), while positive immigrants were in $17 \%(36 / 214)$ cases and in $2 \%(3 / 172)$ controls (aOR 9.6; $95 \% \mathrm{Cl}: 2.9-32.4$ ). Factors associated with a higher risk of infection for all study participants were eosinophilia $(p<0.001)$ and immigration $(p=0.001)$. Overall, strongyloidiasis was nine-times more frequent in individuals with eosinophilia than in those with normal eosinophil count.

\section{Introduction}

Strongyloides stercoralis is a soil-transmitted helminth affecting millions of people worldwide $[1,2]$. Its transmission occurs in areas where poor hygienic conditions and humid, warm climate permit the free-living cycle of the parasite. The larvae present in the soil can penetrate human skin, therefore barefoot walking and agricultural activities pose people at risk of acquiring the infection. S. stercoralis produces larvae that can reinfect the host by a so called auto-infective cycle, a peculiarity shared only by Capillaria spp [3], so that an infected person remains infected life-long, if not properly treated [4]. This is the reason why strongyloidiasis can be diagnosed in people who have left endemic countries already several years before.

The few studies conducted in the United States (US) and in Europe to evaluate the prevalence of strongyloidiasis in immigrants and refugees from endemic countries, either through population or hospital-based studies, probably underestimated the real burden of the infection as long as microscopic stool examination was the only test used for screening [5]. In fact, the methods commonly employed for stool microscopy such as formalin-ether concentration, have a low sensitivity. Preferred faecal-based methods for the detection of S. stercoralis are Baermann funnel concentration and agar plate culture (APC), but the method that has so far demonstrated the highest sensitivity is serology [6]. Studies conducted in the field, classically underestimate the burden of strongyloidiasis if there is no special focus on this infection i.e. through using an appropriate diagnostic method. This is why the 'old' estimates of prevalence from the late $1980 \mathrm{~s}$ and $1990 \mathrm{~s}$ $[7,8]$ were recently questioned $[1,2]$.

The transmission of strongyloidiasis occurs especially in tropical and subtropical areas. However, in some temperate countries, autochthonous transmission occurred in the past $[9,10]$, or might be still ongoing 
Map of northern Italy showing where participating sites are situated, study of Strongyloides stercoralis epidemiology, northern Italy, February 2013-July 2014

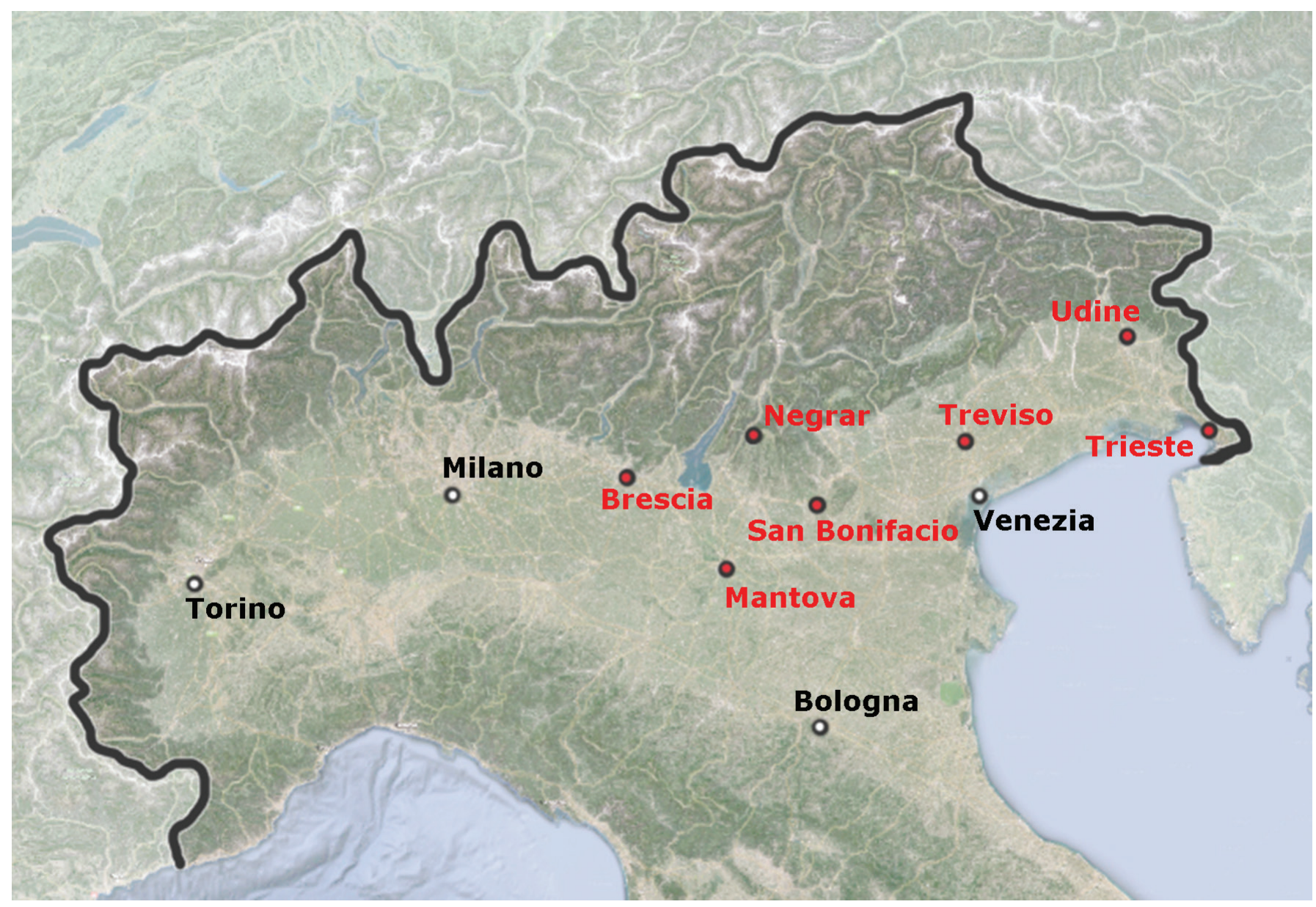

The names of the study sites are in red.

$[11,12]$. Therefore, cases of $S$. stercoralis infection can be diagnosed in people who have never moved from the Mediterranean coast.

Strongyloidiasis can be fatal in immunocompromised patients so prompt diagnosis and effective treatment are crucial for all those infected, in order to prevent later complications, such as disseminated strongyloidiasis [4]. Chronic infection is characterised by mild, unspecific symptoms such as pruritus, abdominal pain or discomfort, respiratory impairment which are not easily attributable to $S$. stercoralis and there is no full agreement among experts on considering eosinophilia as a predictor of the infection [13]. However, in nonendemic countries a high eosinophil count might be a sufficient index of suspicion in travellers or in patients over 65 years with history of barefoot walking in a formerly endemic area $[14,15]$.

The treatment of choice for strongyloidiasis is ivermectin that has demonstrated a higher efficacy than albendazole [16]. Although the drug is included in the
World Health Organization (WHO) list of essential medicines [17], it is not accessible for the vast majority of infected people in the world $[18,19]$. In fact, this essential drug is still donated to endemic countries, but with the strict limitation of use for Wuchereria bancrofti and Onchocerca volvulus control programmes [20]. In Italy, ivermectin has never been registered for human use.

In a previous pilot study, we screened 132 Italian individuals born in 1940 or earlier, with eosinophilia and no significant travel history, presenting to the clinical laboratories of two health districts. The serology test, an in-house immunofluorescence antibody test (IFAT), was positive in $28 \%$ of cases, suggesting that strongyloidiasis can be a relevant cause of eosinophilia in this group of individuals [9].

In the present study, we extended the previous screening in order to estimate the prevalence of strongyloidiasis in six provinces of three Italian Regions. The population analysed included both adult 


\section{FIGURE 2}

Flowchart for inclusion of participants in study of Strongyloides stercoralis epidemiology, northern Italy, February 2013-July 2014

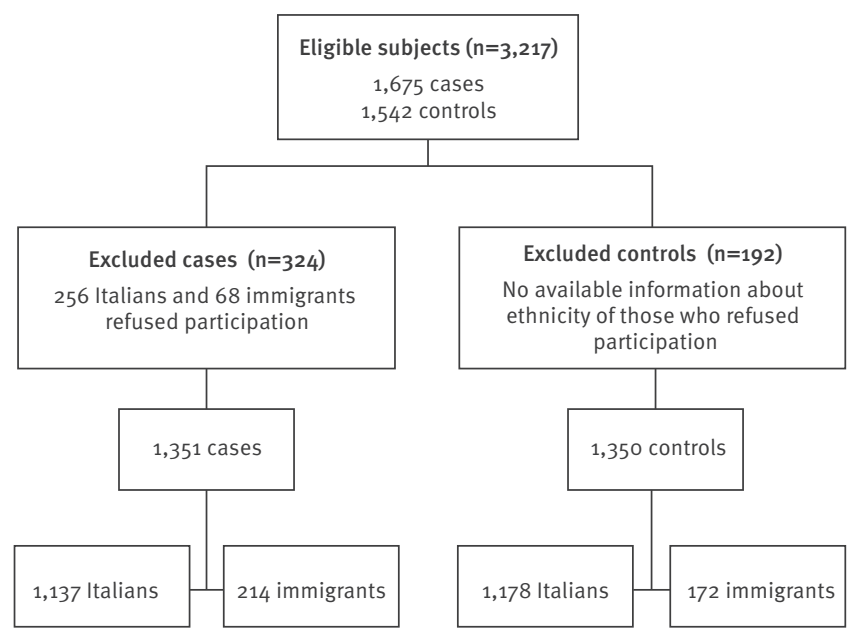

immigrants and Italians born before 1952, with or without eosinophilia.

\section{Methods}

Study design and setting

We conducted a multicentre case-control study.

Participants were enrolled between 2 February 2013 and 27 July 2014. The enrolling sites were the outpatient blood sampling sectors of seven hospitals located in three Italian Regions: Veneto (Negrar, San Bonifacio, and Treviso sites), Lombardia (Brescia, Mantova sites), and Friuli Venezia Giulia (Trieste, Udine sites) (Figure 1). The Centre for Tropical Diseases of Negrar (CTD) and the Health Prevention Department, Verona, were the coordinating centres.

On 1 January 2013, according to the Italian National Institute of Statistics [21], the total resident population in the six provinces of the three Regions involved in the study was 4,215,423 people $(3,742,724$ Italian and 472,699 foreign residents). With regard to Italian residents, $1,074,367(28.7 \%)$ were $>60$ years (born before 1952), which was the age cut off for inclusion of Italians in the present study. As for immigrants, 351,347 (74.3\%) were $>17$ years old, which was the age criterion for their inclusion in the present study.

\section{Participants}

Investigators proposed the screening to individuals meeting the inclusion criteria and consecutively presenting as outpatients to perform a full blood count to one of the collaborating laboratories, during 20 randomly-selected weeks. For the study purpose, we adopted the following definitions:
- Cases: individuals with peripheral eosinophil count $\geq 500 / \mathrm{mcL}$;

- Controls: individuals with eosinophil count<500/ $\mathrm{mcL}$;

- Italians: individuals born and resident in Italy;

- Immigrants: individuals who were born in an endemic area and resided there for at least the first two years of life, and without Italian citizenship.

Each selected week, every centre had to recruit 10 cases and 10 controls. Inclusion criteria were: Italians born before 1952 (as in CTD experience with hundreds of patients the infection was extremely rare in younger Italian individuals with no travel history), immigrants aged $\geq 18$ years. Each participant gave informed written consent. Individuals included in the study received a copy of the result of the test(s) performed and, in case of positive or uncertain result, a treatment with ivermectin $(200 \mu \mathrm{g} / \mathrm{kg}$, stat dose) was offered. A case report form (CRF) with essential clinical data was filled for those with positive test results.

\section{Laboratory methods}

Screening for $S$. stercoralis infection was performed with a commercial ELISA test, IVD Research, CA, USA ELISA (IVD ELISA) during 2013, then Bordier ELISA until the end of the study period, due to unavailability of the former test; positive samples were tested with an in-house IFAT [22]. Discordant samples were analysed with Bordier ELISA during 2013, until when IVD ELISA was used as the screening test. Subsequently, due to unavailability of the latter test, Bordier ELISA was used as the screening test, and a third testing for discordant samples was no longer possible. The three tests have been described in detail elsewhere [23]. Patients testing positive in the screening tests were invited to supply a faecal sample for Koga agar plate culture for S. stercoralis [24] and/or for copro-parasitological test (formalin-ether concentration).

For the study purpose, patients were defined as 'positive' in case of two concordant positive serologic tests and/or a positive screening test AND a positive APC / copro-parasitological test. Individuals with only one positive screening test and negative stool test were classified as 'uncertain' in case a third serologic test was unavailable.

\section{Study size}

There is scarce data on the prevalence of strongyloidiasis in Italy. Previous, smaller studies, found a prevalence between 10 and $15 \%$ in Italians with eosinophilia aged $>60$ and $>68$ years, respectively, and around $4 \%$ in controls of the same age group with normal eosinophil count $[9,14]$. On the basis of these surveys, the study size was calculated considering an odds ratio (OR) for suspected/confirmed strongyloidiasis in cases vs controls of 3, a case/control ratio of 1:1, a prevalence 


\section{FIGURE 3}

Percentage of positivity for Strongyloides stercoralis in relation to eosinophil count in Italians and in immigrants, study of S. stercoralis epidemiology, northern Italy, February 2013-July $2014(\mathrm{n}=2,701)$

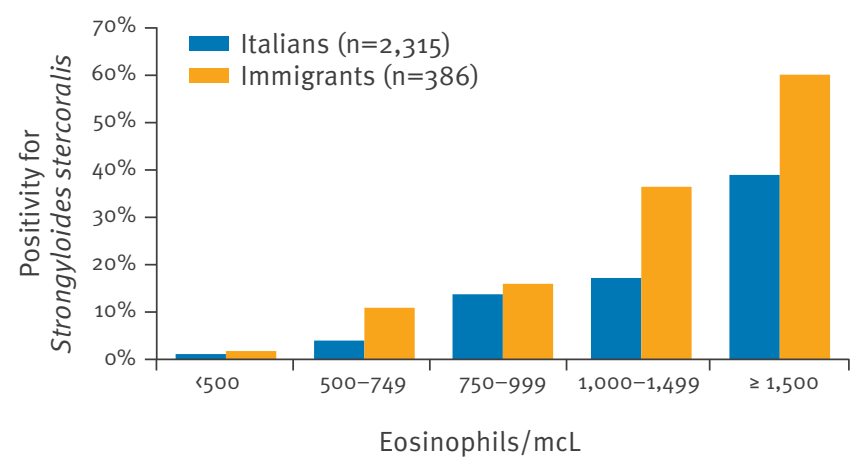

of strongyloidiasis in the control group of $3 \%$, a confidence level at $95 \%$, a study power of $80 \%$ and a design effect of 1.5. Eventually, a total of 950 Italians were to be tested, 475 cases and controls, respectively. Therefore, we initially established to enroll at least 500 individuals per group $(1,000$ Italian individuals in total).

The literature demonstrates a high variability in the prevalence of strongyloidiasis in immigrants, depending on their country of origin and on the screening method used [5]. Studies based on serology demonstrated a prevalence between 10 and $36 \%$, irrespective of the eosinophil count. To calculate the study size we assumed an OR (for strongyloidiasis in cases vs controls) of 3 and a study power $80 \%$. Based on these data, the minimum number of immigrants to be tested was 185 for each group, resulting in a total number of 370. Therefore, we attempted to enroll 200 individuals per group, 400 immigrants in total.

Overall, the minimal sample size required was of 1,400 individuals. As the sample size calculation was based on very weak estimates, particularly for Italian individuals for whom no formal, previous prevalence study was available, the proposed target sample was twice as large i.e. 2,800 individuals, 200 cases and 200 controls to be recruited by each study site.

\section{Statistical methods}

Data at each centre were entered in a pre-structured Excel file and analysed using Stata 10 software (Stata Corp., Texas, US). For quantitative variables, data distribution was checked for normality by Shapiro-Francia test.

Since data were not normally distributed, they were analysed using the non-parametric Mann-Whitney test and the variations among groups were calculated as medians with interquartile ranges (IQR). Associations among categorical variables were analysed by Pearson's chi-squared test or Fisher's exact test as appropriate, and presented as observed frequencies and proportions. Trend analysis was performed by chi-squared test for linear trend. The OR of finding the outcome of interest (i.e. S. stercoralis infection) in relationship to the eosinophil count (defining cases and controls) and to other variables of interest (sex, age, recruitment site, geographical area of origin) were calculated by logistic regression. For all tests, the level chosen to indicate statistical significance was $p<0.05$ (two-tailed).

\section{Ethical issues}

The Ethics Committee of the coordinating centres (Comitato Etico della Provincia di Verona) approved the study protocol on 17 January 2012. The study protocol was then submitted to the Ethics Committees of each of the study sites, and formally approved.

All study participants received an information sheet and a letter for their general practitioner, explaining aim and methods of the study; signed informed consent form was required.

\section{Results}

\section{Participants}

A total of 3,217 individuals fulfilled the inclusion criteria; 516 were not included in the study because they were unable to give informed consent or refused to participate. The total number of individuals included in the study and analysed was 2,701 (Figure 2).

The study population comprised 1,392 men (52\%) and 1,309 (48\%) women. Each participating centre recruited ca 400 individuals.

Among 2,315 Italians, the proportion of women was $41 \%$ $(n=464)$ for the 1,137 cases and $53 \%(n=625)$ for the 1,178 total controls. Median age was 73 years (range: 61-99; IQR: 67-78) and 72 years (range: 61-94; IQR: 67-77) for cases and controls, respectively. Median value of eosinophil count was $630 / \mathrm{mcL}$ (range: $500-$ 24,890; IQR: 550-790) and 150/mcL (range: 0-490; IQR: 100-220) for cases and controls, respectively.

Among 386 immigrants, women represented $48 \%$ $(n=103)$ of the 214 cases, and the proportion was higher for the 172 controls, 68\% $(n=117)$. Median age was 38 years (range: $18-87$; IQR: $30-48$ ) and 40 years (range: 18-83; IQR: 29-53) for cases and controls, respectively. Median value of eosinophil count was 655/mcL (range: 500-2,380; IQR: 570-830) and 145/ mcL (range: 0-460; IQR: 70-240) for the cases and the controls, respectively.

Immigrants originated from Europe, especially eastern Europe and the Balkans (26\%, $n=101)$, Asia (22\%, $n=83)$, Sub-Saharan Africa $(21 \%, n=82)$, North Africa and Middle East (18\%, $n=68)$, and Latin America (13\%, $n=52)$. 
Signs and symptoms compatible with strongyloidiasis in individuals testing positive who answered a questionnaire, study of Strongyloides stercoralis epidemiology, northern Italy, February 2013-July $2014(\mathrm{n}=54)$

\begin{tabular}{|l|c|c|c|}
\hline Signs and symptoms & $\begin{array}{c}\text { Number of Italians (\%) } \\
\mathrm{n}=43\end{array}$ & $\begin{array}{c}\text { Number of immigrants (\%) } \\
\mathrm{n}=11\end{array}$ & $\begin{array}{c}\text { Total number (\%) } \\
\mathrm{n}=54\end{array}$ \\
\hline Pruritus & $23(53.5)$ & $4(36.4)$ & $27(50)$ \\
\hline Skin rash & $13(30.2)$ & $2(18.2)$ & $15(27.8)$ \\
\hline Respiratory symptoms & $16(37.2)$ & $3(27.3)$ & $19(35.2)$ \\
\hline Abdominal pain & $9(20.9)$ & $1(9.1)$ & $10(18.5)$ \\
\hline Diarrhoea & $1(2.3)$ & $2(18.2)$ & $3(5.6)$ \\
\hline
\end{tabular}

\section{Prevalence in cases and in controls}

Overall, of 2,701 participating individuals, 149 (5\%) were classified as positive (110 Italians and $39 \mathrm{immi}$ grants) and 32 (1\%) as uncertain (29 Italians and 3 immigrants).

Among Italians with eosinophilia (cases) 8\% (97/1,137) were positive vs $1 \%(13 / 1,178)$ without eosinophilia. Considering a total population of the same age group of $1,074,367$ in the six provinces, and an average of $4 \%$ of subjects of the same age with eosinophilia (data not shown), we obtain a rough estimate of 4,000 Italians over 60 years of age with S. stercoralis infection.

Among immigrants, positive cases were $17 \%(36 / 214)$ vs $2 \%$ positive controls ( $3 / 172)$, respectively. The proportion of positives was significantly higher among cases, both for Italians $(p<0.001)$ and immigrants ( $p<0.001)$. Moreover, the higher the eosinophil count, the higher was the proportion of infected individuals, in both groups. Among Italians, the proportion of positive individuals ranged from $4 \%$ (31/780) for those with eosinophil counts between 500 and $749 / \mathrm{mcL}$, to $39 \%$ $(21 / 54)$ for those with eosinophil counts $\geq 1,500 / \mathrm{mcL}$ ( $p<0.001$ ) (Figure 3).

Among immigrants, this proportion ranged from $11 \%(15 / 138)$ for those with eosinophils between 500 and $749 / \mathrm{mcL}$, to $60 \%(6 / 10)$ for those with eosinophils $\geq 1,500 / \mathrm{mcL}$ ( $p<0.001$ ) (Figure 3), albeit numbers were small in the latter group. Moreover, among the Italian cases, the proportion of positive individuals showed an upward trend with increasing age $(p<0.001)$ and varied depending on the study site $(p=0.01)$, with a peak in individuals born before 1936 (46/380;12\%) and in those recruited in the sites located in agricultural regions of the Po valley (e.g. San Bonifacio site: $19 / 146 ; 13 \%)$.

Immigrant cases had the following distribution, according to the region of origin of the patients: Latin America (6/31), Sub-Saharan Africa (14/48), Asia (11/55), Europe $(2 / 44)$, and North Africa (3/36).

Some of the individuals who were positive in the screening test refused to provide a stool sample, therefore the results of stool tests were available only for $70 \%(104 / 149)$ of patients with positive serology of which $28 \%(n=29 / 104)$ had a positive stool result. Ninety-nine of 149 positive patients (66\%), plus seven individuals with uncertain result, received ivermectin treatment, offered free of charge to all eligible patients. Information about possible risk factors for complicated strongyloidiasis was available for $83 \%(124 / 149)$ positive individuals: $16 \%(20 / 124)$ presented a current or past condition considered to constitute a risk for the development of severe strongyloidiasis. In the latter group, most (17/20) were treated, while two individuals refused and one died of metastatic breast cancer soon after being tested.

Analysis on the subgroup of 54 of 149 positive individuals who answered the questionnaire showed that the majority had signs and symptoms compatible with strongyloidiasis (Table 1) and had been exposed to a risk factor for infection (farm work 32/54; walking barefoot in earlier years 37/54). Only two of 43 responding Italians reported a stay longer than one month in endemic countries, where they might have had contact with contaminated soil. The remaining Italians did not present a relevant travel history, so we assume that the infection was probably acquired in Italy.

By logistic regression, eosinophilia $(p<0.001)$ and immigration $(p=0.001)$ were independent risk factors for infection for all participants. After adjusting for birth cohort, sex and site of recruitment for Italians, or age, sex and geographical area of origin for immigrants, presence of eosinophilia $\geq 500 / \mathrm{mcL}$ was significantly associated with infection both in Italians (adjusted OR: 8.18; 95\% Cl: 4.53-14.76; p<0.001) and in immigrants (aOR: 9.62; 95\% Cl: 2.85-32.41; $\mathrm{p}$ <0.001). Among Italians, year of birth and site of recruitment maintained a significant association with infection also at the multivariate analysis (Table 2); the same occurred among immigrants with regard to area of origin (Table 3 ).

\section{Discussion}

The high number of screened individuals, especially Italians, in our study, permitted to obtain a valuable estimate of the prevalence of strongyloidiasis in the 


\section{TABLE 2}

Logistic regression analysis of factors associated with Italians testing positive for Strongyloides stercoralis, study of $S$. stercoralis epidemiology, northern Italy, February 2013-July $2014(\mathrm{n}=2,315$ of which 1,137 cases and 1,178 controls)

\begin{tabular}{|l|c|c|c|}
\hline Factors & OR & $95 \% \mathrm{Cl}$ & P value \\
\hline Eosinophil count $\geq 500 / \mathrm{mcL}$ & 8.18 & $4.53-14.76$ & $<0.001$ \\
\hline Sex (male vs female) & 0.93 & $0.63-1.39$ & 0.730 \\
\hline Year of birth \\
\hline $1947-1951$ & 1.00 & Reference & NA. \\
\hline $1937-1946$ & 2.56 & $1.24-5.28$ & 0.011 \\
\hline 1936 or before & 3.95 & $1.90-8.20$ & $<0.001$ \\
\hline Recruitment site & 1.00 & Reference & NA \\
\hline Trieste & 1.34 & $0.54-3.32$ & 0.52 \\
\hline Udine & 1.67 & $0.69-4.00$ & 0.25 \\
\hline Negrar & 2.33 & $1.00-5.41$ & 0.050 \\
\hline Mantova & 2.47 & $1.07-5.70$ & 0.033 \\
\hline Brescia & 2.97 & $1.33-6.64$ & 0.008 \\
\hline Treviso & 3.43 & $1.54-7.68$ & 0.003 \\
\hline San Bonifacio &
\end{tabular}

$\mathrm{Cl}$ : confidence interval; NA: not applicable; OR: odds ratio.

studied regions in the north of Italy: 8 and $17 \%$, respectively, in Italians and immigrants with eosinophilia, 1 and $2 \%$ in those with a normal eosinophil count, irrespective of signs/symptoms of the infection. This finding is relevant for autochthonous Italians, for whom prevalence data were previously limited and patchy, and this study demonstrated a considerable proportion of infected individuals. In addition, $2 \%$ of Italian controls without eosinophilia with positive/uncertain test result is worth of note. The findings indicate that the infection is not an extinguished problem among elderly Italians living in the study areas.

The geographical pattern of infection prevalence is consistent with a higher transmission in agricultural areas of Po valley during the first decades of the past century, with a downward trend over time likely due to improvement of hygiene and sanitary conditions. Parts of the country, in the centre and in the south, presented in the past characteristics that make a location suitable for the free-living cycle of $S$. stercoralis. It is thus probable that a similar epidemiological picture might be prevalent in a large part, if not in the whole, of Italy. This could also be true for other countries in the Mediterranean basin, where sporadic autochthonous strongyloidiasis cases have been diagnosed $[10,12]$.

Among immigrants, the proportion of positive individuals was high among cases with eosinophil counts $\geq 500 /$ $\mathrm{mcL}$, particularly if individuals originated from SubSaharan Africa, Asia, and Latin America.

Prevalence data are fundamental to implement screening and prevention programmes. We believe our results support the establishment of risk categories for screening individuals at risk of developing strongyloidiasis, such as elderly Italians (and, probably, Europeans from other Mediterranean countries) and immigrants with eosinophilia. In the latter group, it might be even costeffective to treat all patients without testing [4]. This should, however, be demonstrated by a well-designed study, also considering that a pre-treatment diagnostic evaluation (obligatorily including serology) is crucial to monitor cure at follow-up [25].

One in three of the infected individuals refused the treatment that was offered free of charge after a thorough explanation of the risk associated with untreated, chronic infection. Even general practitioners were not always keen to collaborate. Our experience suggests that strongyloidiasis is not always perceived as a relevant health problem, not only by the general population, but also by the medical community. To overcome this problem, it would be advisable to create national guidelines for the screening and management of eosinophilia that should consider strongyloidiasis among the differential diagnoses. Moreover, considering that strongyloidiasis can be fatal in immunocompromised individuals, S. stercoralis should be included in guidelines/protocols for screening of candidate patients for immune-suppressant therapies, such as the oncological and rheumatological ones.

\section{Limitations}

We faced some difficulties in finding eligible immigrants for inclusion in the study, therefore the number of immigrants enrolled was slightly lower than planned. This was the reason, in addition to that provided in the Methods part, to recruit a higher number of Italians than the initially calculated sample size, as we did not deem it appropriate, to stop the recruitment in this group and continue only with immigrants. We did not include in the analysis the countries of origin as numbers for such analysis were too small and instead we analysed the continents/macro-areas. We still believe the results are useful, considering the paucity of similar data in the literature. Although the included individuals were not randomly extracted from the general population, the enrolment of out-patients, coming to the hospital laboratory to perform a very simple and common test (full blood count), results in a sample that can be comparable to the general population in that age range, in particular for the larger Italian group. The controls were unmatched, but consecutively recruited on a randomly selected day on a 1:1 basis, within the same main group (Italian or immigrant) and age range.

Finally, the accuracy of serology is high, but false-positive and false-negative results can occur [23]. The use of a second, confirmatory serological test in addition to the faecal-based tests, when available, was aimed to increase the specificity of the results. Sensitivity can be lower in immunocompromised individuals [26], however, we believe that this may have had a minimal influence on the overall results, given the high number 


\section{TABLE 3}

Logistic regression analysis of factors associated with testing positive for Strongyloides stercoralis among immigrant individuals, study of $S$. stercoralis epidemiology, northern Italy, February 2013-July 2014 $(\mathrm{n}=386$ of which 214 cases and 172 controls)

\begin{tabular}{|c|c|c|c|}
\hline Factors & OR & $95 \% \mathrm{Cl}$ & $P$ value \\
\hline Eosinophil count $\geq 500 / \mathrm{mcL}$ & 9.62 & $2.85-32.41$ & $<0.001$ \\
\hline Sex (male vs female) & 1.70 & $0.82-3.54$ & 0.16 \\
\hline Age (+1 year) & 1.00 & $0.97-1.03$ & 0.83 \\
\hline \multicolumn{4}{|l|}{ Geographical area of origin } \\
\hline Europe & 1.00 & Reference & NA \\
\hline North Africa/Middle East & 1.77 & $0.28-11.27$ & 0.55 \\
\hline Asia & 5.01 & $1.02-24.59$ & 0.047 \\
\hline Latin America & 6.33 & $1.20-33.40$ & 0.030 \\
\hline Sub-Saharan Africa & 9.54 & $2.01-45.19$ & 0.004 \\
\hline
\end{tabular}

$\mathrm{Cl}$ : confidence interval; NA: not applicable; OR: odds ratio.

of individuals screened [19]. The screening test had to be changed, however Bordier ELISA and IVD demonstrated similar accuracy in our previous study [23]. Therefore, we believe that the number of patients positive at screening might not have been substantially different with IVD ELISA. On the other hand, this change entailed the lack of a third serology test, therefore patients with discordant results had to be classified as uncertain. PCR was not available at our Centre before 2014, hence we could not use this method, that showed good accuracy compared with APC and Baermann technique $[27,28]$. PCR is less cumbersome than the traditional faecal-based methods and the samples can be stored (either frozen or with ethanol), therefore it could have been a useful tool considering the high number of individuals screened.

\section{Conclusions}

The improvement of hygienic conditions and sanitation, and the availability of deworming drugs are likely to successfully control most helminth infections in endemic areas in the forthcoming years. However, the lack of mass drug administration programmes specifically targeting $S$. stercoralis (using ivermectin) might lead to long-term persistence of this infection in some individuals. It is also important to note that, due to the peculiarity of the auto-infective cycle of S. stercoralis, this parasite may remain once the other helminth infections have disappeared. This has been observed in Italy. Physicians should be aware of the categories of patients that would require screening for S. stercoralis infection.

\section{Members of the CCM Strongyloides Study Group}

Fabrizio Abrescia, Alessandra Arzese, Mara Baldissera, Matteo Bassetti, Zeno Bisoffi, Dora Buonfrate, Silvio Caligaris, Giacomo Caramaschi, Francesco Castelli, Stefano Ciaffoni, Paola Coato, Mario Giobbia, Maria Gobbo, Patrizia Guzzo, Roberto Luzzati, Franco Manzato, Marta Mascarello,
Cristina Maurel, Maria Merelli, Giovanna Muffato, Giuseppina Napoletano, Elena Pasqualini, Chiara Postiglione, Antonio Regazzoli, Paola Rodari, Novella Scattolo, Pier Giorgio Scotton, Anna Turrini, Gianluca Zulian.

\section{Acknowledgements}

The study received financial support from the Italian Ministry of Health, under the CCM framework (National Centre for Prevention and Control of Diseases).

We warmly thank Stefania Bonafini, Monica Degani, Tiziana Serafin and Stefano Tais for the fundamental technical support.

\section{Conflict of interest}

None declared.

\section{Authors' contributions}

D Buonfrate, Z Bisoffi, and M Baldissera drafted the manuscript. Z Bisoffi and $F$ Abrescia conceived the study. D Buonfrate, Z Bisoffi, F Abrescia and G Napoletano coordinated the investigations and supervised the study. M Baldissera performed the statistical analysis. M Bassetti, F Castelli, N Scattolo were responsible for the coordination of local investigations. Epidemiological investigations were conducted by D Buonfrate, M Mascarello, M Giobbia, G Caramaschi. All authors commented and agreed upon the final manuscript.

\section{References}

1. Schär F, Trostdorf U, Giardina F, Khieu V, Muth S, Marti H, et al. Strongyloides stercoralis: Global Distribution and Risk Factors. PLoS Negl Trop Dis. 2013;7(7):e2288. DOI: 10.1371/ journal.pntd.0002288 PMID: 23875033

2. Bisoffi Z, Buonfrate D, Montresor A, Requena-Méndez A, Muñoz J, Krolewiecki AJ, et al. Strongyloides stercoralis: a plea for action. PLoS Negl Trop Dis. 2013;7(5):e2214. DOI: 10.1371/journal.pntd.0002214 PMID: 23675546

3. Dronda F, Chaves F, Sanz A, Lopez-Velez R. Human intestinal capillariasis in an area of nonendemicity: case report and review.Clin Infect Dis. 1993:17(5):909-12. DOI: 10.1093/ clinids/17.5.909 PMID: 8286640

4. Mejia R, Nutman TB. Screening, prevention, and treatment for hyperinfection syndrome and disseminated infections caused by Strongyloides stercoralis.Curr Opin Infect Dis. 2012;25(4):458-63. DOI: 10.1097/QCO.obo13e3283551dbd PMID: 22691685

5. Buonfrate D, Angheben A, Gobbi F, Muñoz J, RequenaMendez A, Gotuzzo E, et al. Imported strongyloidiasis: epidemiology, presentations, and treatment. Curr Infect Dis Rep. 2012;14(3):256-62. DOI: 10.1007/s11908-012-0248-6 PMID: 22322601

6. Requena-Mendez A, Chiodini P, Bisoffi Z, Buonfrate D Gotuzzo E, Munoz J. The laboratory diagnosis and follow up of strongyloidiasis: a systematic review. PLoS Negl Trop Dis. 2013;7(1):e2002.

7. Genta RM. Global prevalence of strongyloidiasis: critical review with epidemiologic insights into the prevention of disseminated disease. Rev Infect Dis. 1989;11(5):755-67. DOI: 10.1093/clinids/11.5.755 PMID: 2682948

8. Jorgensen T, Montresor A, Savioli L. Effectively controlling strongyloidiasis.Parasitol Today. 1996;12(4):164. DOI: 10.1016/0169-4758(96)80806-4 PMID: 15275229

9. Abrescia FF, Falda A, Caramaschi G, Scalzini A, Gobbi F, Angheben A, et al. Reemergence of strongyloidiasis, northern Italy. Emerg Infect Dis. 2009;15(9):1531-3. DOI: 10.3201/ eid1509.090191 PMID: 19788836

10. Martinez-Perez A, Lopez-Velez R. Is strongyloidiasis endemic in Spain?PLoS Negl Trop Dis. 2015;9(2):e0003482. DOI: 10.1371/ journal.pntd.0003482 PMID: 25654324

11. Russell ES, Gray EB, Marshall RE, Davis S, Beaudoin A, Handali $S$, et al. Prevalence of Strongyloides stercoralis antibodies 
among a rural Appalachian population--Kentucky, 2013. Am J Trop Med Hyg. 2014;91(5):1000-1. DOI: 10.4269/ajtmh.14-0310 PMID: 25157122

12. Duvignaud A, Pistone T, Malvy D. Strongyloidiasis in a young French woman raises concern about possible ongoing autochthonous transmission in Spain.Int J Infect Dis. 2016;42:43-4. DOI: 10.1016/j.ijid.2015.11.015 PMID: 26617257

13. Requena-Mendez A, Buonfrate D, Bisoffi Z, Munoz J. Advances in the diagnosis of human strongyloidiasis.Curr Trop Med Rep. 2014;1(4):207-15. DOI: 10.1007/S40475-014-0034-7

14. Pirisi M, Salvador E, Bisoffi Z, Gobbo M, Smirne C, Gigli C, et al. Unsuspected strongyloidiasis in hospitalised elderly patients with and without eosinophilia. Clin Microbiol Infect. 2006;12(8):787-92. DOI: 10.1111/j.1469-0691.2006.01500.x PMID: 16842575

15. Checkley AM, Chiodini PL, Dockrell DH, Bates I, Thwaites GE, Booth HL, et al. , British Infection Society and Hospital for Tropical Diseases. Eosinophilia in returning travellers and migrants from the tropics: UK recommendations for investigation and initial management.J Infect. 2010;60(1):1-20. DOI: 10.1016/j.jinf.2009.11.003 PMID: 19931558

16. Suputtamongkol Y, Premasathian N, Bhumimuang K, Waywa D, Nilganuwong S, Karuphong E, et al. Efficacy and safety of single and double doses of ivermectin versus 7-day high dose albendazole for chronic strongyloidiasis. PLoS Negl Trop Dis. 2011;5(5):e1044. DOI: 10.1371/journal.pntd.0001044 PMID: 21572981

17. World Health Organization (WHO). WHO Model List of Essential Medicines. Geneva: WHO. Apr 2015. Available from: http:// www.who.int/medicines/publications/essentialmedicines/en/

18. Krolewiecki AJ, Lammie P, Jacobson J, Gabrielli AF, Levecke $B$, Socias E, et al. A public health response against Strongyloides stercoralis: time to look at soil-transmitted helminthiasis in full. PLoS Negl Trop Dis. 2013;7(5):e2165. DOI: 10.1371/journal.pntd.0002165 PMID: 23675541

19. Anselmi M, Buonfrate D, Guevara Espinoza A, Prandi R, Marquez M, Gobbo M, et al. Mass Administration of Ivermectin for the Elimination of Onchocerciasis Significantly Reduced and Maintained Low the Prevalence of Strongyloides stercoralis in Esmeraldas, Ecuador. PLoS Negl Trop Dis. 2015;9(11):e0004150. DOI: 10.1371/journal.pntd.0004150 PMID: 26540412

20. World Health Organization (WHO). Preventive chemotherapy in human helminthiasis. Coordinated use of anthelminthic drugs in control interventions: a manual for health professionals and programme managers. Geneva. WHO. 2006. Available from: http://apps.who.int/iris/ bitstream/10665/43545/1/9241547103_eng.pdf

21. Italian National Institute of Statistics. Rome. [Accessed 12 Jan 2016]. Available from: www.istat.it

22. Boscolo M, Gobbo M, Mantovani W, Degani M, Anselmi $M$, Monteiro GB, et al. Evaluation of an indirect immunofluorescence assay for strongyloidiasis as a tool for diagnosis and follow-up. Clin Vaccine Immunol. 2007;14(2):129-33. DOI: 10.1128/CVI.00278-06 PMID: 17135451

23. Bisoffi Z, Buonfrate D, Sequi M, Mejia R, Cimino RO, Krolewiecki AJ, et al. Diagnostic accuracy of five serologic tests for Strongyloides stercoralis infection. PLoS Negl Trop Dis. 2014;8(1):e2640. DOI: 10.1371/journal.pntd.0002640 PMID: 24427320

24. Koga K, Kasuya S, Khamboonruang C, Sukhavat K, leda $M$, Takatsuka $N$, et al. A modified agar plate method for detection of Strongyloides stercoralis. Am J Trop Med Hyg. 1991;45(4):518-21.PMID: 1951861

25. Buonfrate D, Sequi M, Mejia R, Cimino RO, Krolewiecki AJ, Albonico M, et al. Accuracy of five serologic tests for the follow up of Strongyloides stercoralis infection. PLoS Negl Trop Dis. 2015;9(2):e0003491. DOI: 10.1371/journal.pntd.0003491 PMID: 25668740

26. Mascarello M, Gobbi F, Angheben A, Gobbo M, Gaiera G, Pegoraro $M$, et al. Prevalence of Strongyloides stercoralis infection among HIV-positive immigrants attending two Italian hospitals, from 2000 to 2009. Ann Trop Med Parasitol. 2011;105(8):617-23. DOI: 10.1179/2047773211Y.0000000006 PMID: 22325821

27. Becker SL, Piraisoody N, Kramme S, Marti H, Silué KD, Panning $M$, et al. Real-time PCR for detection of Strongyloides stercoralis in human stool samples from Côte d'lvoire: diagnostic accuracy, inter-laboratory comparison and patterns of hookworm co-infection. Acta Trop. 2015;150:210-7. DOI: 10.1016/j.actatropica.2015.07.019 PMID: 26215130

28. Saugar JM, Merino FJ, Martín-Rabadán P, Fernández-Soto $P$, Ortega S, Gárate T, et al. Application of real-time PCR for the detection of Strongyloides spp. in clinical samples in a reference center in Spain. Acta Trop. 2015;142:20-5. DOI: 10.1016/j.actatropica.2014.10.020 PMID: 25447829

\section{License and copyright}

This is an open-access article distributed under the terms of the Creative Commons Attribution (CC BY 4.0) Licence. You may share and adapt the material, but must give appropriate credit to the source, provide a link to the licence, and indicate if changes were made.

This article is copyright of the authors, 2016. 